\title{
Desarrollo de una actividad experiencial para la enseñanza de estadística.
}

\section{Ángel Peiró-Signes ${ }^{\mathrm{a}}$, Oscar Trull-Domínguez ${ }^{\mathrm{b}}$ y Marival Segarra-Oña ${ }^{\mathrm{c}}$}

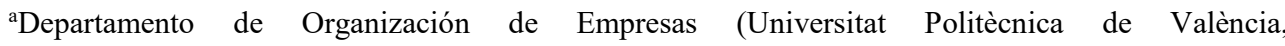
anpeisig@omp.upv.es), bDepartamento de Estadística e Investigación Operativa Aplicada y Calidad (Universitat Politècnica de València, otrull@eio.upv.es) y ${ }^{\mathrm{c} D e p a r t a m e n t o ~ d e ~ O r g a n i z a c i o ́ n ~ d e ~ E m p r e s a s ~}$ (Universitat Politècnica de València, maseo@omp.upv.es).

\begin{abstract}
This paper describes how to apply an experiential learning activity to show the basis of statistical concepts applied in the industry. The activity simulates a real application of a packaging line in an randomly selected production period. The activity allows the students to share an experience to build on top further superior leanings. This paper develops the experience with the instructions to garatee its application and efficacy in the class session. Preliminary results on the preceptions of the students about the contents treated on the session are encouraging.
\end{abstract}

Keywords: experiential learning, critical thinking, simulation, statistics

\begin{abstract}
Resumen
Este artículo describe como aplicar una actividad de aprendizaje experiencial para ilustrar las bases de los conceptos estadísticos aplicados en la industria. La actividad simula una aplicación real de una linea productiva de empaquetado en un periodo de tiempo aleatorio de producción. La actividad permite a los alumnos compartir una experiencia que sobre los que se puede construir aprendizajes de nivel superior. Este artículo desarrolla la experiencia con instrucciones para garantizar la correcta aplicación y eficacia en la sesión de aula. Los resultados preliminares de la percepción de los estudiantes sobre los contenidos son esperanzadores.
\end{abstract}

Palabras clave: Aprendizaje experiencial, pensamiento crítico, simulación, estadística.

\section{Introducción}

A la hora de planificar los profesores tienen en mente varios aspectos. Por una parte, están los aspectos determinados por los condicionantes de los planes de estudios, es decir, contenidos y competencias que deben trabajarse. Por otro lado, encontramos otros aspectos relacionados con el desarrollo del curso como la participación, la motivación del alumnado o la capacidad de las actividades propuestas para conseguir los objetivos planteados. 
Este artículo presenta una actividad de aprendizaje experiencial para ilustrar algunos de los conceptos fundamentales de la estadística. El objetivo central de esta experiencia es exponer a los alumnos a una serie de aspectos de estadística de gran aplicación para la gestión de los procesos en la industria. De forma más concreta, los conceptos de media, desviación típica, variabilidad, número de defectos y capacidad de los procesos. La experiencia nos servirá de iniciador para explorar conceptos más complejos de tipo estadístico que pueden aplicarse en el ámbito industrial.

\section{Objetivos}

Según LaForge y Busing (1998), toda actividad de aprendizaje experiencial sigue una serie de pasos que van desde la fase de planificación, pasando por las fases de introducción y ejecución de la actividad, para finalizar en la fase de retroalimentación.

Este artículo tiene como objetivo desarrollar y testar una actividad de aprendizaje experiencial perfilando todas las fases de la experiencia. La fase de planificación es crucial para el éxito de la actividad. Comprende el establecimiento de objetivos y la determinación de las bases, normas y recursos necesarios para desarrollar la actividad, se determina la forma de gestionar el desarrollo de la actividad y la fase final de discusión y retroalimentación.

La dificultad a la que se enfrenta el profesor a la hora de diseñar tiene que ver con la capacidad de crear una actividad, que sin llegar a la complejidad de la realidad productiva, refleje de forma relevante los aspectos importantes que se quieren desarrollar en el curso (Halpern y Hakel, 2003). Asimismo, la actividad debe permitir a los estudiantes enfrentarse a situaciones que requieran tomar decisiones durante el proceso. Buscamos también al final de la experiencia un proceso de reflexión sobre aquellos aspectos que han emergido durante la actividad. De esta forma, mediante la reflexión crítica se consigue una mayor significancia del aprendizaje (Kolb, 1984; Wheeler y McLeod, 2002).

En definitiva, esta actividad pretende ir más allá del mero conocimiento de algunos conceptos básicos de estadística y del análisis de datos. Enlaza la estadística con situaciones del mundo productivo industrial.

Por otro lado, uno de los aspectos más complicados de la tarea docente tiene que ver con la motivación del alumno o, al menos, la no desmotivación del mismo. Para mantener el interés del alumno, los profesores utilizan distintas estrategias que normalmente involucran de forma más activa al alumno en las sesiones. Las actividades de aprendizaje experiencial han demostrado su capacidad y eficacia a la hora de dinamizar el trabajo del aula (Peiro-Signes and Segarra-Oña, 2015), incluso en distintos niveles educativos (Peiro-Signes, 2015). La actividad de aprendizaje experiencial presentada en este artículo pretende también mantener interés y participación del alumno en la sesión de aula y, simultáneamente, trabajar algunas capacidades de tipo transversal que son de gran importancia para el desarrollo de los futuros graduados.

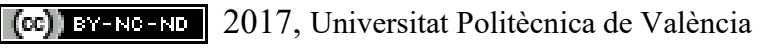




\section{Desarrollo de la innovación.}

La primera fase del desarrollo es la planificación. Para poder planificar debemos establecer claramente cuales van a ser los objetivos de aprendizaje. En un temario típico de estadística podemos encontrar objetivos como: identificar, conocer, representar y describir distribuciones de variables aleatorias - tanto discretas como continuas - determinando sus parámetros de posición, dispersión y forma; realizar inferencias sobre la población de distribuciones y aplicarlos al control de calidad; etc.

Un aspecto clave a la hora de planificar es establecer la conexión entre los objetivos de aprendizaje del asignatura y la actividad. Esta conexión es lo que va a permitir la percepción por parte del alumno de la actividad como útil en lugar de una pérdida de tiempo.

Esta actividad está ligada con los objetivos antes comentados de: conocer conceptos básicos de estadística, reconocer su aplicación a situaciones reales, comprender la importancia del concepto de variabilidad y sus consecuencias, y analizar la capacidad de los procesos industriales. Consiste en la simulación de un proceso industrial de envasado en bolsas. Los alumnos son divididos en grupos que simularán este proceso industrial. Después de una breve introducción donde se dan las instrucciones, cada grupo, con los materiales y recursos establecidos, deberá llenar bolsas de plástico. Tras unos minutos de producción, la producción para y se inicia la fase de reflexión y discusión. Para ello, se plantean una serie de preguntas a los grupos para estimular la discusión acerca de los aspectos que han ocurrido durante la experiencia. Finalmente, el facilitador de la actividad realiza una recapitulación de lo ocurrido para ayudar a los estudiantes a entender los conceptos de estadística involucrados en una actividad típica industrial, el uso que se puede dar a los datos y análisis realizados y las posibles formas de mejorar los resultados a partir de dicho análisis. Esta actividad está planteada para una duración de entre 40 y 50 minutos, dependiendo en el nivel del curso.

\section{a. Introducción}

Para preparar la actividad es importante crear cierta curiosidad en el alumnado. Para ello, con anterioridad a la entrada de los alumnos, el facilitador debería de tener preparada el aula con grupos de mesas y todos los materiales que cada grupo va a utilizar. A la entrada de los alumnos en el aula, atendiendo al tamaño de la clase, el instructor formará grupos de tres a cinco alumnos. Los grupos deben formarse rápidamente para centrar la atención en la actividad. Una vez los alumnos están divididos en grupos y situados en sus mesas el instructor debe de establecer las reglas del ejercicio.

\section{b. Desarrollo}

Cada grupo dispondrá de una caja con alubias blancas donde selectivamente se han puesto algunas alubias pintas, un vaso donde se ha realizado una marca a lo largo de todo su perímetro para marcar hasta donde hay que llenar cada bolsa, bolsitas transparentes de plástico, una báscula electrónica, una hoja de toma de datos y un bolígrafo.

El proceso de producción comienza una vez el instructor ha distribuido a los equipos en las mesas y ha dado las instrucciones. Cada equipo tiene entre 5 y 10 minutos para formar paquetes de alubias blancas mediante un medidor volumétrico (un vaso). El tiempo exacto se 
ajusta para conseguir que todos los grupos dispongan de, al menos, 50 datos. Cada grupo debe de llenar el vaso hasta la marca indicada para conseguir la cantidad de alubias que debe ir en cada bolsa. Cada bolsa medida será pesada en una báscula y se anotará su peso exacto con dos decimales en la hoja de recogida de datos. Para poner un poco de presión, similar al que puede haber en el mundo industrial, mediremos también la productividad de los equipos. Cada minuto que pase durante la actividad será marcado por el instructor y los responsables de cada equipo deberán anotar en la hoja de datos el número de bolsas elaboradas hasta el momento. Este apartado nos permitirá posteriormente enriquecer el debate generado confrontando los conceptos de productividad y calidad. Asimismo, dos de los equipos recibirán respectivamente y sin saberlo un vaso con la marca de volumen un poco por debajo del resto y otro con la línea un poco por encima. De esta manera, las medias de datos de estos equipos serán respectivamente inferiores y superiores a la del resto.

\section{c. Discusión y debate}

Al finalizar el periodo marcado, se informa a los alumnos de que el tiempo se ha acabado y se les pedirá que paren inmediatamente de producir más bolsas. En este momento empieza la fase de análisis de los datos y retroalimentación de la experiencia. En esta fase, se recomienda a los alumnos utilizar el ordenador o algún tipo de hoja de cálculo. La primera tarea será calcular los parámetros de centrado y dispersión: media, mediana, rango y desviación típica sobre el peso de las bolsas fabricadas. Los alumnos a partir de los datos deberán también realizar un histograma que les permita ver la distribución de datos. El instructor recoge los datos globales de todos los equipos e incita a comparar los resultados de cada uno de los grupos.

Preguntas como ¿Por qué los datos que obtenemos no son iguales? ¿Qué elementos determinan la distribución de datos en el proceso? ¿Qué equipo produce mejor?¿Qué parámetro tenemos que ver para evaluar la calidad de un proceso productivo?¿Cómo puedo variar la media en el proceso?¿Cómo puedo disminuir la variabilidad del proceso?, son preguntas que pueden dirigir el debate hacia algunos de los aspectos clave que se quieren trabajar en la experiencia: ¿Qué causa la variabilidad? ¿Cómo mejorar la variabilidad de los procesos? ¿Cómo mejorar los resultados de un proceso sin mejorar la variabilidad? ¿Qué relación tiene la variabilidad con el consumo de materiales, las pérdidas o los beneficios en un proceso?

Posteriormente se introduce un límite de peso inferior para las bolsas. Las bolsas deben de pesar, al menos, $\mathrm{x}$ gramos para ser aceptables por nuestro cliente. De forma que los alumnos tienen que calcular que porcentaje de bolsas se encontrarían fuera del peso establecido y proponer medidas para su reducción y/o eliminación. A partir de unos datos sobre el coste de mano de obra, la productividad obtenida, el coste de la bolsa, horas de trabajo,... los alumnos deberán calcular el coste anual de las bolsas que no llegan al peso establecido. De forma similar, a partir de los costes de producción (materia prima, bolsa y mano de obra), tendrán que determinar a partir de qué sobrepeso convendría romper la bolsa para realizar una bolsa nueva.

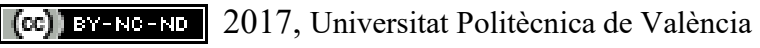


Esta fase del análisis permite a los alumnos aplicar las herramientas estadísticas para tomar decisiones en entornos industriales muy cercanos a la realidad. Para estimular la discusión el instructor puede plantear preguntas como: ¿por qué debe haber un límite de peso inferior? ¿si no existiera ese límite que problemas y podríamos tener con nuestros clientes? ¿qué repercusión tiene la variabilidad en la calidad del producto? ¿y en el coste?.

Finalmente, en la tercera parte del análisis, los alumnos tienen que buscar en las bolsas cuantas judías pintas tienen. Las judías pintas representan defectos. Si en una bolsa hay más de 3 defectos se considera que la bolsa es defectuosa. Este apartado del ejercicio nos permite diferenciar el concepto de defecto y pieza defectuosa, valorar el porcentaje aproximado de defectos en el producto y la probabilidad de bolsas defectuosas que fabricaremos. Preguntas como ¿qué diferencia hay entre defecto y bolsa defectuosa? ¿Qué implicación tiene cada uno de ellos desde el punto de vista del cliente? ¿cómo se puede reducir el número de defectos? ¿Cómo se puede reducir el número de piezas defectuosas?, pueden estimular el debate y la discusión acerca de estos aspectos.

Después de la experiencia los estudiantes deberían de reconocer cómo los conceptos y herramientas estadísticas facilitan la toma de decisiones en la industria, evaluar el impacto cuantitativo y económico de la variabilidad de un proceso o analizar críticamente cuáles pueden ser sus causas y posibles soluciones.

Al final del periodo de retroalimentación y debate, el instructor puede empezar una pequeña lección magistral de los conceptos experimentados de forma más estructurada, que permita a los estudiantes pensar de una forma más profunda sobre lo vivido en la experiencia y llegar a niveles superiores de aprendizaje (Meyers, 1986; Smith, 2003).

\section{Resultados}

Este artículo plantea la utilización de una actividad de aprendizaje experiencial en el aula. El éxito o fracaso de la actividad deberá evaluarse en forma de resultados. Para ello se debe realizar una evaluación de los resultados al final del curso y después de varias aplicaciones. El análisis de los resultados debe ser complementado al final del curso con una evaluación del alcance de los objetivos propuestos tras introducir actividades experienciales adicionales. Para una evaluación preliminar, planteamos una pequeña encuesta mediante una escala a Likert 1 a 5 donde 1 corresponde a totalmente en desacuerdo y 5 a totalmente de acuerdo. Las preguntas de la Figura 1 se plantean en los términos "Si el examen se realizara hoy ¿crees que recibirías la máxima nota en las preguntas siguientes?". Este test permite al instructor evaluar de manera sencilla el impacto del ejercicio y determinar si son necesarios ajustes para conseguir los objetivos propuestos. Los resultados preliminares sobre la primera experiencia (ver figura 1) en un grupo de 25 alumnos son esperanzadores. Los alumnos perciben que su conocimiento sobre los conceptos trabajados en la experiencia son lo bastante buenos como para responder correctamente a las preguntas planteadas en un hipotético examen. Estos resultados, aun conociendo de sus limitaciones, parecen indicar que la experiencia tiene un gran potencial para producir aprendizajes significativos en los estudiantes. 
Explica la diferencia entre defecto y pieza defectuosa

Explica cuando un proceso es capaz y sobre qué elementos deberíamos de trabajar para conseguir que fuera capaz.

Explica cómo puede afectar la variabilidad a la calidad de un producto.

Explica por qué existe variabilidad en los procesos. ¿Qué concepto estadístico está relacionado con la variabilidad?

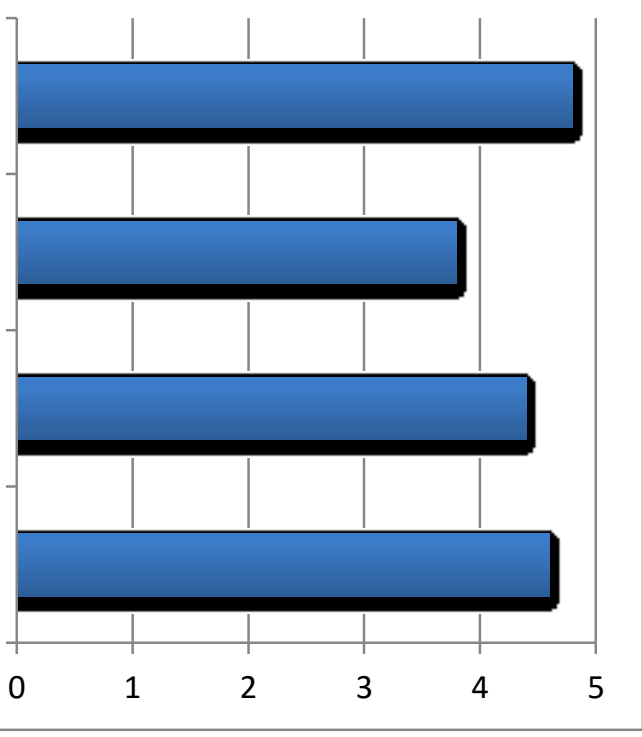

Fig. 1 Resultados medios sobre el cuestionario de evaluación de la actividad.

\section{Conclusiones}

Este artículo presenta una actividad de aprendizaje experiencial diseñada para la enseñanza de conceptos estadísticos. La actividad pretende ayudar a conseguir un aprendizaje más significativo y relacionado con las actividades futuras de los egresados de asignaturas de grado en el área industrial y de ingeniería. Esta experiencia cubre aspectos fundamentales de la recogida, cálculo y presentación de datos estadísticos así como, del análisis y evaluación para la mejora en la toma de decisiones. En este artículo se muestran las numerosas posibilidades que tiene la experiencia para relacionar conceptos y visualizar aspectos complejos de los entornos productivos que sería difícil cubrir mediante metodologías docentes tradicionales. Adicionalmente, la percepción de los alumnos sobre la retención de los conceptos tratado es elevada tras la experiencia lo que anima a utilizar esta metodología frente a otras tradicionales que aparentemente parecen menos eficaces.

\section{Agradecimientos}

Este trabajo forma parte del estudio desarrollado por el Grupo de Aprendizaje Experiencial (GAE) creado como EICE en la Universidad Politécnica de Valencia (UPV). Los autores desean agradecer a la UPV por el apoyo a través del PIME 2017 " Adaptación y desarrollo de aprendizajes experienciales al contexto de las asignaturas ".

\section{Referencias}

HALPERN, D. y HAKEL, M. (2003). "Applying the science of learning”. Change, vol. 35, issue 4, p. 36-41. 
KOLB, D. (1984). Experiential learning: Experience as the source of learning and development.

Englewood Cliffs, NJ: Prentice Hall.

LAFORGE, R. y BUSING, M. (1998). "The use of industrial software to create experiential learning activities in operations management courses". Production and Operations Management, vol. 7, issue 3, p. 325-334.

MEYERS, C. (1986). Teaching students to think critically. San Francisco, CA: Jossey-Bass.

PEIRO-SIGNES, A., SEGARRA-OÑA, M., DE MIGUEL-MOLINA, M., J. ALBORS-GARRIGÓS, J. y DE-MIGUEL-MOLINA B. (2015). "The experiential learning activity: benefits and difficulties in real learning environments". Proceedings of ICERI2015 Conference, p. 7231-7237.

PEIRO-SIGNES, A. y SEGARRA-OÑA, M.; (2015). "Experiential learning as a dynamizer of class activity”. Proceedings of ICERI2015 Conference, p. 1010-1013.

SMITH, G. (2003). "Beyond critical thinking and decision making: Teaching business students how to think". Journal of Management Education, vol. 27, issue 1, p. 24-51.

WHEELER, J., y MCLEOD, P. (2002). "Expanding our teaching effectiveness: Understanding our responses to in-the-moment classroom events". Journal of Management Education, vol. 26, issue 6, p. 693-716. 